\title{
The effects of sodium pentobarbital on matching and oddity performance in pigeons
}

\author{
ANGELO SANTI \\ Wilfrid Laurier University, Waterloo, Ontario N2L 3C5, Canada
}

\begin{abstract}
The effects of sodium pentobarbital on matching and oddity performance in pigeons were examined by employing a higher-order conditional discrimination paradigm. In this paradigm, the line orientation which was superimposed on all of the response keys signaled whether a response to the matching color or a response to the nonmatching color was correct. All pigeons had extensive previous training in this paradigm and were tested at each of three dosage levels: $5,7.5$, and $10 \mathrm{mg} / \mathrm{kg}$. For all birds, a clear dose-related decrease in accuracy was observed; however, the effect was not differential for matching and oddity trials. Accuracy reductions were accompanied by an increase in position preference on both types of trials. The data are compatible with recent claims that physical identity of the sample and correct comparison stimulus need have no special status for pigeons.
\end{abstract}

In a matching-to-sample paradigm, the correct response is the selection of the comparison stimulus which is identical to the sample stimulus. In an oddity-from-sample paradigm, it is the comparison stimulus which is different from the sample stimulus that is correct. Hence, the assignment of the correct comparison stimulus (SD) is based on identity for matching but is arbitrary for oddity. In a standard three-key matching-to-sample paradigm, pigeons acquire a set of SD rules (Berryman, Cumming, Cohen, \& Johnson, 1965; Cumming, Berryman, \& Cohen, $1965)$ such that each sample designates one or more comparison stimuli as correct. With three sample stimuli, oddity mastery would require two SD rules for each sample stimulus (e.g., if red, select green; if red, select blue), while matching would require only one SD rule for each sample (e.g., if red, select red). Cumming and Berryman (1965) utilized three sample stimuli and found that, for pigeons, matching was acquired faster and had a higher terminal level of accuracy than oddity. This difference was apparently due to the greater number of SD rules which oddity subjects had to acquire. When the number of SD rules required for acquisition of matching and oddity are equivalent, the rates of acquisition are similar (Zentall \& Hogan, 1974).

\footnotetext{
This research was supported by Grant A9801 from the National Research Council of Canada to Jo Tombaugh and by a National Research Council Postgraduate Scholarship to the author. Portions of this research were presented at the meeting of the Canadian Psychological Association, Vancouver, 1977. I would like to thank Jo Tombaugh, Hymie Anisman, and Evanne Woods for their assistance, as well as this journal's reviewers for their helpful comments and suggestions. Reprints are available from the author, Department of Psychology, Wilfrid Laurier University, Waterloo, Ontario N2L 3C5, Canada.
}

The physical identity between sample and correct comparison stimulus in a matching paradigm apparently need have no special status for pigeons. This has been established by comparisons of matching-tosample and symbolic matching-to-sample (Carter \& Eckerman, 1975; Cohen, Looney, Brady, \& Aucella, 1976). In symbolic matching-to-sample, the sample bears an arbitrary relation to comparison stimuli since they are typically on different dimensions (e.g., colored samples with line comparisons). Carter and Eckerman (1975) demonstrated that rates of acquisition for hue-line and line-line matching (where the first term designates the sample stimulus dimension and the second term the comparison dimension) differed as a function of the discriminability of one sample from another, while identity between sample and comparison stimuli played no role for pigeons in speed of acquisition. Cohen et al.(1976) found no differences in the rates and patterns of acquisition of both matching and symbolic matching when pigeons were pretrained on a successive discrimination with the two stimuli that were to be used as samples and had differential sample response requirements during matching-to-sample training.

Santi (1978) trained pigeons in a higher-order conditional discrimination paradigm in which the line orientation which was superimposed on all response keys signaled whether a response to the matching color or a response to the nonmatching color (i.e., oddity) was correct. For half of the birds, a $0^{\circ}$ line was correlated with reinforcement for matching reponses, while a $90^{\circ}$ line was correlated with reinforcement for oddity responses. This relationship was reversed for the remaining birds. Following training, stimulus control gradients were obtained by varying 
the angularity of the lines. For each bird, as the orientation correlated with reinforcement for matching responses on the color dimension was varied, there was a decreasing tendency to select the matching comparison. In addition, responding in the presence of a line tilt, which was intermediate between the line tilt signaling matching trials and the line tilt signaling oddity trials, failed to reveal a bias toward or away from the physically identical comparison stimulus.

While these data suggest that physical identity of the sample and correct comparison stimuli need have no special status for pigeons, there remains some anomalous data. These data derive from studies by Berryman, Cumming, Nevin, and Jarvik (1964) and Nevin and Liebold (1966). Both of these studies demonstrated that, with increased doses of sodium pentobarbital, matching accuracy was reduced to a greater extent than oddity accuracy. These results are difficult to interpret, since they seem to contradict the general finding that more "complex" discriminations are more easily disrupted by drugs (Dews, 1955). However, they do suggest that the assignment of an SD on the basis of identity might result in different processes being operative than when an SD is assigned arbitrarily. Recently, Eckerman, Lanson, and Berryman (1978) have examined the effects of pentobarbital on symbolic matching and symbolic oddity performance in pigeons. They found equivalent disruption on both types of tasks, even though the symbolic oddity task had more SD rules than the symbolic matching task. This indicated that the number of SD rules learned was probably not a reason for the differential drug effects previously reported by Berryman et al. (1964). A further analysis of just those symbolic matching or symbolic oddity trials that were equivalent to simple matching and simple oddity trials indicated a tendency for greater disruption on simple matching trials, but the trend was not statistically significant. The failure to find a significant differential effect of pentobarbital in this study indicates that the prior findings may not represent a robust phenomenon. In order to pursue the question further, the present study was undertaken to provide a direct within-subject comparison of the effects of sodium pentobarbital on matching and oddity performance in pigeons.

\section{METHOD}

\footnotetext{
Subjects

Six White Carneaux pigeons, with unrestricted access to water and grit in their home cages, were maintained at $80 \%$ of their free-feeding weights throughout the experiment. Prior to the present experiment, all subjects had received higher-order conditional discrimination training as well as transfer testing on the color dimension and stimulus control testing on the line-tilt dimension (cf. Santi, 1978).
}

\section{Apparatus}

Four Lehigh Valley Model 1519 pigeon chambers were used. Each chamber was enclosed in a plywood box insulated with acoustical ceiling tile. Masking noise and ventilation were provided by two 100-cfm Dayton blowers located on the left side wall and back wall, respectively, of each apparatus and holding box. The three translucent (Perspex) response keys were all $2.54 \mathrm{~cm}$ in diameter. The stimuli used (red, green, yellow, or blue and a white line with an orientation of $0^{\circ}, 22.5^{\circ}, 45^{\circ}, 67.5^{\circ}$, or $90^{\circ}$ from horizontal) could be projected on the back of the translucent keys by an In-Line Digital Unit. Directly below the center key was a $5.08-\mathrm{cm}$ square opening that provided access to a hopper filled with mixed grain. General illumination was provided by a houselight located above the center response key. All experimental contingencies and response measures were programmed and recorded by a PDP-8I digital computer located in a separate room.

\section{Procedure}

Higher-order conditional discrimination paradigm. Red or green lights were presented with a line tilt of $0^{\circ}$ or $90^{\circ}$ superimposed on them. One of the side keys was illuminated with a color that was the same as that on the center key, while the other side key was illuminated with a color different from that on the center key. The line orientation which was superimposed on all response keys signaled whether a response to the matching color or a response to the nonmatching color was correct. For three of the birds (P1, P5, and P7), the $0^{\circ}$ line was correlated with reinforcement for responding to the color which matched that on the center key, while the $90^{\circ}$ line was correlated with reinforcement for responding to the color which did not match that on the center key. For the remaining three birds (P4, P6, and P8), this relationship was reversed. Since the red and green stimuli were counterbalanced over the left and right keys and the superimposed line tilt was $0^{\circ}$ or $90^{\circ}$, eight different stimulus configurations resulted.

Sessions consisted of 144 trials (18 randomized blocks of the 8 different stimulus combinations). A trial commenced with the illumination of the center key by a line tilt superimposed on a colored background. A response to this key resulted in the presentation of the appropriate comparison stimuli on both of the side keys. A peck to the correct comparison stimulus was followed by 3-sec access to grain, while a peck to the incorrect comparison was followed by a 3-sec blackout (all lights in the experimental chamber were turned off). Following either reinforcement or blackout, an intertrial interval of $15 \mathrm{sec}$ occurred, during which only the houselight was illuminated.

\section{Drug Assessment}

Sodium pentobarbital was dissolved in $.9 \%$ sodium chloride solution and prepared so that the volume injected by weight was always $1.0 \mathrm{ml} / \mathrm{kg}$ of body weight. The dosages $(5,7.5$, and $10 \mathrm{mg} / \mathrm{kg}$ ) were administered in a mixed order with five repetitions of each dose. Drug sessions alternated with saline control sessions. Either the drug or saline was injected into the pectoral muscle $10 \mathrm{~min}$ before each session.

\section{RESULTS}

The relationship between accuracy on matching and oddity trials as a function of the various dosages of sodium pentobarbital is shown in Figure 1. In this figure, the data have been averaged over sessions at each dosage level. Under saline conditions, accuracy is high on both matching and oddity trials. For all six pigeons, a dose-related decrease in accuracy can 


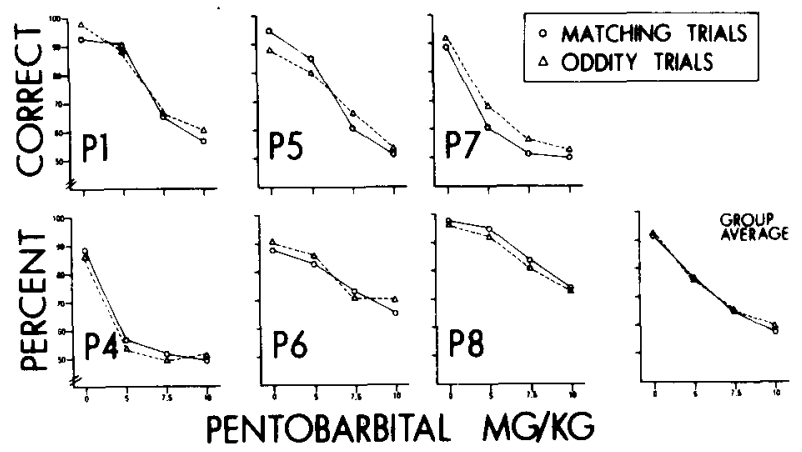

Figure 1. Percent correct on matching and oddity trials as a function of dosage of sodium pentobarbital for each bird.

be observed which is not differential for matching and oddity trials. These data were submitted to an analysis of variance appropriate for the two withinsubject variables (trial type and drug dosage). The analysis indicated a significant main effect for drug dosage $[F(3,35)=21.01, p<.01]$. Application of the Newman-Keuls test indicated that all pairwise comparisons of the various dosage levels were significant except for the difference between 7.5 and $10.0 \mathrm{mg} / \mathrm{kg}$. Neither trial type nor the Trial Type by Drug Dosage interaction was significant $(F<1)$.

In order to clarify the source of the accuracy reductions, a more detailed analysis was undertaken. This is presented in Figure 2, which shows accuracy of responding as a function of the position of the correct comparison stimulus, drug dosages, and trial blocks within sessions. These data indicate that accuracy reductions are primarily a reflection of increased position preferences and that within-session recovery of accuracy is obtained, especially at lower dosage levels. For example, the data of P7 reveal that under saline, accuracy was very high regardless of the position of the correct comparison stimulus. Under $5 \mathrm{mg} / \mathrm{kg}$ of pentobarbital, accuracy was markedly reduced on the right key during the first third of the experimental session and then gradually improved. Under $7.5 \mathrm{mg} / \mathrm{kg}$ of pentobarbital, accuracy was further reduced on the right key and the within-session recovery was small. Under $10 \mathrm{mg} / \mathrm{kg}, \mathrm{P} 7$ was responding almost exclusively to the left key throughout the entire session, so that accuracy on the right key was less than $10 \%$ and no recovery occurred within sessions. The other birds showed similar effects.

It was noted that pigeons in this matching-to-sample paradigm would peck at the sample stimulus more than once on some trials even though only one peck was required to produce the comparison stimuli. In five of the six birds, the number of these extra centerkey pecks increased as a function of increasing concentration of the drug.

\section{DISCUSSION}

The present results replicate the finding that sodium pentobarbital will reduce the accuracy of matching-to-sample performance. However, they do not replicate the differential sensitivity for matching and oddity which has previously been reported (Berryman et al., 1964; Nevin \& Liebold, 1966). The present data are more compatible with the drug effects reported by Eckerman et al. (1978) and with recent evidence which suggests that physical identity of the sample and correct comparison stimulus need have no special status for pigeons (Carter \& Eckerman, 1975; Cohen, et al., 1976; Santi, 1978). A number of procedural factors can be ruled out as contributing to the previously reported differential drug effects on matching and oddity tasks. Eckerman et al. (1978) have shown that the number of SD rules does not necessarily contribute to differential drug effects. The use of a between-subject as opposed to within-subject design also does not appear to be critical, since nondifferential drug effects have been obtained in both the Eckerman et al. (1978) betweensubject study and the present within-subject study.

One factor which may be important in accounting for the discrepant results is the nature of drug administration. In the Berryman et al. (1964) study, matching subjects were given progressively increased doses of pentobarbital over sessions, while oddity subjects were given a random order of dose levels. It is known that repeated administration of barbiturates, such as pentobarbital, gradually increases the amount of hepatic enzymes responsible for degrading these drugs (Iversen \& Iversen, 1975). This can result in the drug being more rapidly degraded and its action diminished with repeated administration. The

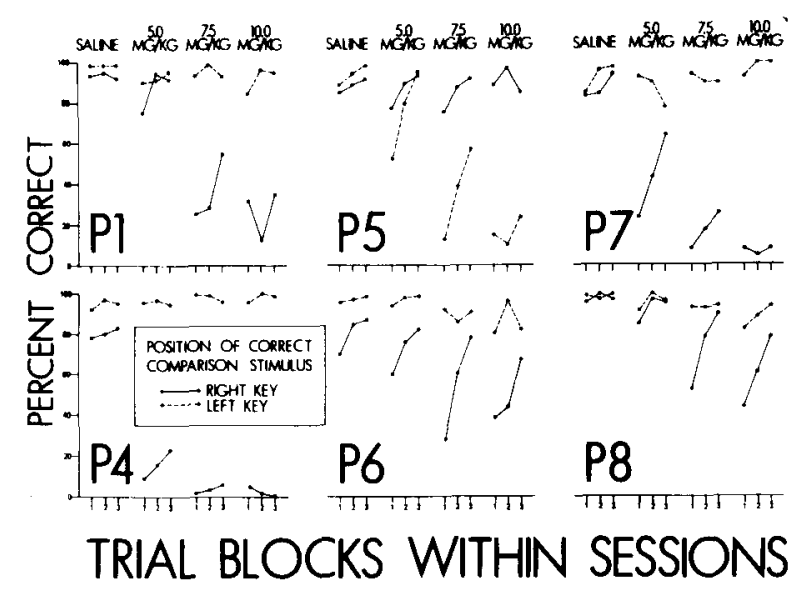

Figure 2. Percentage of correct responding as a function of drug dosage, position of the correct comparison stimulus, and trial blocks within sessions for each bird. The data are based on both the matching and the oddity trials. 
different methods of administering the doses to oddity and matching subjects could have resulted in differences in the activity of hepatic-drug metabolizing enzymes between the two groups of birds in the Berryman et al. (1964) study. The results of the Nevin and Liebold (1966) study cannot be accounted for in terms of differences in drug administration, but it is unclear how much emphasis should be placed on their results, since they were based only on a single subject.

The mechanism that underlies the effect of sodium pentobarbital on matching behavior is not entirely understood. It is known that barbiturates possess a particular ability to depress the function of the reticular formation (Himwich \& Rinaldi, 1957). In addition, there has been recent behavioral confirmation of a hypothesis, proposed by Pragay \& Mirsky (1973), that barbiturates impair the discriminative performance of monkeys by retarding the rate at which sensory stimuli can be processed and utilized (Bartus \& Johnson, 1977). The sensory processing deficits in monkeys are probably a result of altered reticular formation functioning. It may be that in pigeons, as well, pentobarbital retards the mechanisms involved in processing visual stimuli. The increase in extra center-key pecks to sample stimuli found in this study may represent an attempt to compensate for these deficits.

\section{REFERENCES}

Bartus, R. T., \& Johnson, H. R. Primate information processing under sodium pentobarbital and chlorpromazine: Differential drug effects with tachistoscopically presented discriminative stimuli. Psychopharmacology, 1977, 53, 249-254.

Berryman, R., Cumming, W. W., Cohen, L. R., \& Johnson, D. F. Acquisition and transfer of simultaneous oddity. Psychological Reports, 1965, 17, 767-775.
Berryman, R., Cumming, W. W., Nevin, J. A., \& Jarvik, M. E. Effects of sodium pentobarbital on complex operant discriminations. Psychopharmacologia, 1964, 6, 388-398.

Carter, D. E., \& Eckerman, D. A. Symbolic matching by pigeons: Rate of learning complex discriminations predicted from simple discriminations. Science, 1975, 187, 662-664.

Cohen, L. R., Looney, T. A., Brady, J. H., \& Aucella, A. F. Differential sample response schedules in the acquisition of conditional discriminations by pigeons. Journal of the Experimental Analysis of Behavior, 1976, 26, 301-314.

Cumming, W. W., \& Berryman, R. The complex discriminated operant: Studies of matching-to-sample and related problems. In D. I. Mostofsky (Ed.), Stimulus generalization. Stanford, Calif: Stanford University Press, 1965.

Cumming, W. W., Berryman, R., \& Cohen, L. R. Acquisition and transfer of zero-delay matching. Psychological Reports, $1965,17,435.445$.

DEws, P. B. Studies on behavior: II. The effects of pentobarbital, methamphetamine and scopolamine on performances in pigeons involving discriminations. Journal of Pharmacology and Experimental Therapeutics, 1955, 115, 380-389.

Eckerman, D. A., Lanson, R. N., \& Berryman, R. Effects of sodium pentobarbital on symbolic matching and symbolic oddity performance. Bulletin of the Psychonomic Society, 1978, 11, 171-174.

Himwich, H. E., \& Rinaldi, F. The effect of drugs on the reticular formation. In W. S. Fields (Ed.), Brain mechanism and drug action. Springfield: Thomas, 1957.

Iversen, S. D., \& Iversen, L. L. Behavioral pharmacology. New York: Oxford University Press, 1975.

Nevin, J. A., \& Liebold, K. Stimulus control of matching and oddity in a pigeon. Psychonomic Science, 1966, 5, 351-352.

Pragay, E. B., \& Mirsky, A. F. The nature of performance deficit under secobarbital and $\mathrm{CPZ}$ in the monkey. Psychopharmacologia (Berlin), 1973, 28, 73-85.

SANTI, A. The role of physical identity of the sample and correct comparison stimulus in matching-to-sample paradigms. Journal of the Experimental Analysis of Behavior, 1978, 29, 511-516.

Zentall, T., \& Hogan, D. Abstract concept learning in the pigeon. Journal of Experimental Psychology, 1974, 102, 393.398.

(Received for publication February 22, 1978; revision accepted September 9, 1978.) 